\title{
GPR183 Gene
}

National Cancer Institute

\section{Source}

National Cancer Institute. GPR183 Gene. NCI Thesaurus. Code C101352.

This gene is involved in humoral immunity. 